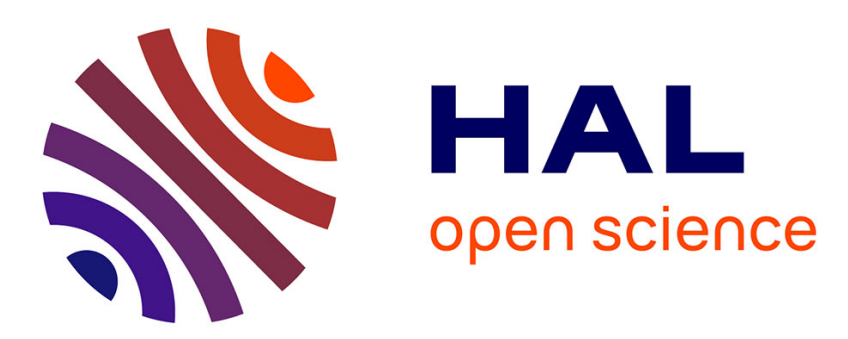

\title{
On the completeness problem for fractional rationals with incommensurable differentiation orders
}

\author{
Hüseyin Ackçay, Rachid R. Malti
}

\section{To cite this version:}

Hüseyin Ackçay, Rachid R. Malti. On the completeness problem for fractional rationals with incommensurable differentiation orders. 17th IFAC World Congress, Jul 2008, Séoul, South Korea. pp.100. hal-00355514

\section{HAL Id: hal-00355514 \\ https://hal.science/hal-00355514}

Submitted on 22 Jan 2009

HAL is a multi-disciplinary open access archive for the deposit and dissemination of scientific research documents, whether they are published or not. The documents may come from teaching and research institutions in France or abroad, or from public or private research centers.
L'archive ouverte pluridisciplinaire HAL, est destinée au dépôt et à la diffusion de documents scientifiques de niveau recherche, publiés ou non, émanant des établissements d'enseignement et de recherche français ou étrangers, des laboratoires publics ou privés. 


\title{
On the Completeness Problem for Fractional Rationals with Incommensurable Differentiation Orders
}

\author{
Hüseyin Akçay* Rachid Malti** \\ * Department of Electrical and Electronics Engineering, Anadolu \\ University, 26470 Eskişehir, Turkey (Tel: +90222 3350580 -X 6459; \\ e-mail: huakcay@anadolu.edu.tr). \\ ** IMS, UMR 5218 CNRS, Université Bordeaux1, 351 cours de la \\ Libération, F 33405 Talence cedex, France (E-mail: \\ rachid.malti@laps.ims-bordeaux.fr).
}

\begin{abstract}
In this paper, completeness problem for a class of fractional rational basis functions with incommensurable differentiation orders is studied. Using the Müntz-Szász theory, it is established that the completeness problem for this class of the basis functions is equivalent to another completeness problem for a particular set of uncountably many basis functions. This equivalence allows one to draw fairly general conclusions on the nature of the completeness problem for fractional rational basis functions with incommensurable differentiation orders.
\end{abstract}

Keywords: Fractional calculus; Basis functions; Completeness; Incommensurable.

\section{INTRODUCTION}

The fractional calculus is a generalization of the traditional calculus that leads to similar concepts and tools but with a much wider applicability. The mathematical concept and formalism of fractional calculus originate from the works of Liouville (1832) and Riemann (1892). For almost three hundred years, it has remained an interesting, but abstract, mathematical concept. In recent years, fractional calculus has been taken up by scientists and engineers and applied in an increasing number of fields, namely in the areas of thermal engineering, acoustics, electromagnetism, control, robotics, viscoelasticity, diffusion, turbulence, signal processing, and many others.

There are many linear systems with transfer functions that can be represented as fractional differential systems, that is, as functions $G(s)$ which involve fractional powers of $s$. For instance, in the field of diffusion, recent work by Schneider (1990) generalizes diffusion equations based on noninteger derivatives. A fractal model for anomalous losses in ferromagnetic materials was used in Vorperian (1992). In rheology, stress in a viscoelastic material is proportional to noninteger derivative of deformation Heymans and Bauwens (1994). In signal processing, noninteger derivative is used in the synthesis of fractal noise Mandelbrot and Ness (1968). In thermal diffusion in a semi-infinite homogeneous medium, Battaglia et al. (2001) have shown that exact solution for the heat equation links thermal flux to a half order derivative of the surface temperature on which the flux is applied. Diffusion phenomena were investigated in semi-infinite planar, spherical and cylindrical media by Oldham and Spanier $(1970,1972,1973)$ who showed that the involved transfer functions use the Laplace variable $s$ with exponents which are multiples of 0.5 . In electrochemical diffusion of charges in the electrode and the electrolyte, the most common physical model used in the litterature is the Randles model (Rodrigues et al. (2000)) which uses Warburg impedance which involves an integrator of order 0.5. In the area of control, the idea of using fractional systems for modeling ideal loop transfer functions dates back to Bode (1945). He showed that the loop gain must have a frequency behavior described as a fractional order transfer function to reduce the effects of disturbances and uncertainties on the closed loop system performance. Recently, in Oustaloup and Mathieu (1999) the advantages of a fractional order controller known as commande robust d'ordre non entier (CRONE) with respect to classical devices was shown. Fractional proportional-integral-derivative controller applications are reported in Podlubny (1999). System identification with fractional models was initiated by Lay et al. (1998). An overview of system identification methods based on fractional models is presented in Malti et al. (2006). Recently, Benchellal et al. (2007) have used fractional models to identify thermal diffusive systems.

Of the greatest interest to the signal processing and control engineering communities is the fact that the fractional systems have both short and long term memories. Some basic properties of fractional systems such as stability Matignon (1998), observability and controllability Matignon and D'Andrea-Novel (1996), and the $H_{\infty}$-norm Sabatier et al. (2005) have been investigated over the last ten years.

A fundamental idea in various areas of applied mathematics, control theory, system identification, signal processing, and system analysis is that of decomposing (perhaps infinite dimensional) descriptions of linear-time-invariant dynamics in terms of an orthonormal basis. This approach is of greatest utility when accurate system descriptions are achieved with only a small number of basis functions. 
In recognition of this, there has been much work Mendel (1966); Epstein (1965) over the past several decades and, with renewed interest, more recently Wahlberg and Mäkilä (1996); Heuberger et al. (1995); Ninness et al. (1998) on the construction, analysis, and application of rational orthonormal bases suitable for providing linear system characterizations.

An important motivation for the consideration of orthonormal parametrizations is for approximation purposes. In this setting, a dominant question must arise as the quality of the approximation. Pertaining to this, one of the most fundamental properties that might be required is completeness. In Akçay and Ninness (1999a,b), model sets spanned by fixed pole orthonormal bases were investigated. These bases generalize the well-known Laguerre and Kautz bases. It was shown that the obtained model sets are complete in $H_{2}(\Pi)$, the space of functions which are analytic on the open right-half plane denoted by $\Pi$ and square integrable on the imaginary axis. In contrast to the Laguerre and the Kautz bases, where all the poles are fixed at the same value, this generalization enjoys increased flexibility of pole location. For example, slow and fast modes may coexist in the model structure. As a result, a fewer number of basis functions may be used without sacrificing model accuracy.

The first complete fractional orthonormal basis, the socalled fractional Laguerre basis was synthesized in Aoun et al. (2007). This extension from the rational Laguerre basis to a fractional one provides a new class of fixed denominator models for system approximation and identification. In Malti et al. (2004), a fractional orthogonal Kautz basis was synthesized. The results in Aoun et al. (2007); Malti et al. (2004) were generalized in Akçay (2007), to fractional bases with prescribed basis poles satisfying only a mild condition on the choice of poles.

In all of these works Aoun et al. (2007); Malti et al. (2004); Akçay (2007), the chosen basis functions are rationals in $s^{\gamma}$ for some fixed $\gamma>0$ despite in many applications, incommensurable differentiation orders are used to describe physical laws. For example, interconnection of mechanical networks with dissipative elements whose constituve relations are best described by fractional derivatives leads to fractional rational transfer functions with incommensurable differentiation orders. In Bonnet and Partington (2000), stability and stabilization of incommensurable transfer functions are studied.

This paper is organized as follows. In $\S 2$, mathematical background on fractional derivatives and fractional rational transfer functions is briefly reviewed. In $\S 3$, fractional rational basis functions with incommensurable differentiation orders are introduced and their impulse responses are briefly examined to motivate the subject of this paper. The orthonormalization of the basis functions and the calculation of their impulse responses in closed form are not studied in this paper. The reader is referred to the three-stage scheme and the residue method presented in Akçay (2007). In $\S 4$, completeness problem for the incommensurable basis functions is studied; and using the Müntz-Szász theory, it is established that the completeness problem for the introduced basis functions is equivalent to another completeness problem for a particular class of uncountably many basis functions. This preliminary result allows one to draw fairly general conclusions on the nature of the completeness problem. $\S 5$ concludes the paper.

\section{FRACTIONAL LINEAR SYSTEMS}

In this section, we review definitions and results of fractional calculus pertinent to our completeness study. The readers are referred to Oldham and Spanier (1974); Samko et al. (1993) and the references therein for details.

Let $\mathbf{R}$ and $\mathbf{C}$ denote the sets of the real and the complex numbers, respectively. The set of the negative numbers (including zero) and its complement in $\mathbf{C}$ are denoted respectively by $\mathbf{R}_{-}$and $\mathcal{R}_{0}$. The real and the imaginary parts of $z \in \mathbf{C}$ are denoted respectively by $\operatorname{Re}(z)$ and $\operatorname{Im}(z)$. The inverse Laplace transform of $F(s)$ denoted by $f(t)$ is defined by

$$
f(t)=\frac{1}{2 \pi j} \int_{\sigma-j \infty}^{\sigma+j \infty} F(s) e^{s t} d s \quad(t>0)
$$

where $\sigma \in \mathbf{R}$ is inside the region of convergence. It is related to $F(s)$ by the Laplace transformation:

$$
F(s)=\int_{0}^{\infty} f(t) e^{-s t} d t
$$

Note the following relation Oldham and Spanier (1974):

$$
\int_{0}^{\infty} D_{\mathrm{d}}^{\gamma} f(t) e^{-s t} d t=s^{\gamma} F(s) \quad \text { for } \operatorname{Re}(s)>0
$$

where $D_{\mathrm{d}}^{\gamma} f(t)$ denotes the fractional derivative of order $\gamma$ of $f(t)$.

The multi-valued function $s^{\gamma}$ becomes an analytic function in the complement of its branch cut line as soon as a branch cut line, i.e. $\mathbf{R}_{-}$, is specified. This choice is made for causal systems.

Let us assume that $F(s)$ is a fractional rational transfer function:

$$
F(s)=\sum_{k=1}^{L} \sum_{\ell=1}^{n_{k}} \frac{\alpha_{k, \ell}}{\left(s^{\gamma_{k}}+\lambda_{k}\right)^{\ell}}
$$

for some complex numbers $\alpha_{k, \ell}, \lambda_{k}$, and positive integers $n_{k}$. From (1)-(3), note that $F$ is the transfer function of a linear system described by the fractional differential equation in the form:

$$
\sum_{n=0}^{N} a_{n} D_{\mathrm{d}}^{\tilde{\gamma}_{n}} y(t)=\sum_{m=0}^{M} b_{m} D_{\mathrm{d}}^{\hat{\gamma}_{m}} u(t)
$$

for some differentiation orders $\tilde{\gamma}_{n}, \hat{\gamma}_{m}$; and complex numbers $a_{n}$ and $b_{m}$.

A system with transfer function $F(s)$ is said to be stable if $F$ is a bounded analytic function on $\Pi$. This means that the system defined by (5) maps bounded energy inputs $u(t)$ to bounded energy outputs $y(t)$. In fact, if this happens then (5) maps magnitude bounded inputs to magnitude 
bounded outputs as well, that is, the fractional linear system (5) is bounded-input/bounded-output (BIBO) stable. The fractional system defined by (5) is stable if and only if $\gamma_{k}$ and the argument of $\lambda_{k}$ denoted by $\arg \left(\lambda_{k}\right)$ in (4) satisfy the inequalities

$$
0<\gamma_{k}<2 \text { and }\left|\arg \left(\lambda_{k}\right)\right|<\pi\left(1-\frac{\gamma_{k}}{2}\right) \text { for all } k \text {. }
$$

Henceforth, we will restrict the parameters $\gamma_{k}$ to the interval $(0,2)$. The inequalities (6) are obtained by applying a stability result in Matignon (1998) to simple fractional rationals $\left(s^{\gamma_{k}}+\lambda_{k}\right)^{-1}$. In Matignon (1998), this stability result is derived assuming that the differentiation orders are commensurable. However, this result extends to the incommensurable differentiation order case considered here without modification.

\section{FRACTIONAL ORTHONORMAL BASES}

The expansion (4) of a fractional linear system (5) suggests approximating functions in $H_{2}(\Pi)$ by linear combinations of the functions $\left(s^{\gamma_{k}}+\lambda_{k}\right)^{-\ell}, 1 \leq \ell \leq n_{k} ; k \geq 1$ where the pairs $\left(\gamma_{k}, \lambda_{k}\right)$ satisfy the inequalities in (6). In doing so, there are two degrees of freedom: the choice of the basis poles encoded by the parameters $\lambda_{k}$ and $\gamma_{k}$ and the choice of the pole multiplicities.

In Akçay (2007), $\gamma_{k}$ was fixed as $\gamma$ and $\lambda_{k}$ was varied subject to the stability requirement (6). It was established there that the linear span of the above fractional rationals is dense in $H_{2}(\Pi)$ provided that a mild condition on the parameters $\lambda_{k}$ are satisfied. The purpose of the current paper is to study this completeness problem when $\gamma_{k}$ is changed and $\lambda_{k}$ is held constant as $\lambda$.

To be more specific on this, we define the so-called generator functions as follows

$$
\phi_{k \ell}(s)=\frac{1}{\left(s^{\gamma}+\lambda\right)^{m_{k}}\left(s^{\gamma_{k}}+\mu\right)^{\ell}}, \quad 1 \leq \ell \leq n_{k} ; k \geq 1
$$

where $\gamma \in(0,2), \lambda>0, m_{k} \geq 0$ are convergence factors assuring $\phi_{k \ell}$ are in $H_{2}(\Pi)$ for all $k, \ell$, and the pairs $(\gamma, \lambda)$ and $\left(\gamma_{k}, \mu\right), k=1,2, \cdots$ satisfy $(6)$. The generator functions (7) generalize the fractional Laguerre functions studied in Aoun et al. (2007).

The inner products of the generator functions (7) are defined by

$$
\begin{aligned}
& <\phi_{k_{1} \ell_{1}}, \phi_{k_{2} \ell_{2}}>= \\
& \frac{1}{2 \pi} \int_{-\infty}^{\infty} \frac{\left[(j \omega)^{\gamma}+\lambda\right]^{-m_{k_{1}}}\left[\overline{(j \omega)^{\gamma}}+\bar{\lambda}\right]^{-m_{k_{2}}}}{\left[(j \omega)^{\gamma_{k_{1}}}+\mu\right]^{\ell_{1}}\left[\overline{(j \omega)^{\gamma_{k_{2}}}}+\bar{\mu}\right]^{\ell_{2}}} d \omega
\end{aligned}
$$

where

$$
(j \omega)^{\gamma}=|\omega|^{\gamma} \begin{cases}e^{j \frac{\pi \gamma}{2}}, & \omega>0 \\ e^{-j \frac{\pi \gamma}{2}}, & \omega<0\end{cases}
$$

When $\gamma_{k_{1}} \neq \gamma_{k_{2}}$, the calculation by hand is tedious.

The generator functions (7) are not orthonormal. Their impulse responses are not real-valued either unless $\mu \in \mathbf{R}$. In Akçay (2007), a three-step procedure that ensures realvalued impulse responses of the synthesized orthonormal basis functions with the same linear span of the generator functions are described.

In order to motivate the introduction of the generator functions (7), when $m_{k}=0$ and $\mu \in \mathbf{R}$ consider their impulse responses given by Akçay (2007)

$$
\begin{aligned}
\widehat{\phi}_{k \ell}(t)= & \frac{1}{\pi} \sum_{i=1}^{\ell} \frac{\ell !}{i !(\ell-i) !} \int_{0}^{\infty} \frac{\mu^{\ell-i} \sin \left(\pi \gamma_{k} i\right) x^{\gamma_{k} i} e^{-x t} d x}{\left[x^{2 \gamma_{k}}+2 \mu x^{\gamma_{k}} \cos \left(\pi \gamma_{k}\right)+\mu^{2}\right]^{\ell}} \\
& +\sum_{p=0}^{1 \text { or } 2} \sum_{q=0}^{\ell-1} c_{p q} t^{q} e^{\alpha_{p q} t}, \quad t>0
\end{aligned}
$$

for some complex numbers $c_{p q}, \alpha_{p q}$. The presence of the second term and the range of $p$ as well as the numbers $c_{p q}, \alpha_{p q}$ depend on the values of $\gamma_{k}$ and $\mu$. This term has the character of a linear time-invariant dynamics and quickly dies out since $\operatorname{Re}\left(\alpha_{p q}\right)<0$ for all $p$ and $q$. The first term is more profound. In fact, from the definition of the gamma function:

$$
\Gamma(\beta)=\int_{0}^{\infty} e^{-z} z^{\beta-1} d z
$$

we have as $t \rightarrow \infty$,

$$
\widehat{\phi}_{k \ell}(t) \approx \sum_{i=1}^{\ell} \frac{\ell ! \sin \left(\pi \gamma_{k} i\right) \Gamma\left(\gamma_{k} i+1\right)}{i !(\ell-i) ! \pi \mu^{\ell+i}} t^{-\left(\gamma_{k} i+1\right)} .
$$

Thus, the fractional rationals appear to be more suitable than the rationals in modeling slowly decaying impulse responses. Moreover, as $\gamma_{k}$ varies more flexible model structures are obtained in comparison to model structures that employ a finite set of fractional orders.

\section{MAIN RESULT}

In this section, we will establish the main result of this paper using the Müntz-Szász theory. To this end, first we have the following auxilary result.

Lemma 1. For two given numbers $\alpha, \beta \in(0,2)$, let

$$
d_{\delta}=\inf _{\omega \in \mathbf{R}, z \in \mathcal{C}_{\delta}}\left|(j \omega)^{z}+\mu\right| \quad(\delta>0)
$$

where the pair $(\beta, \mu)$ satisfies $(6)$ and

$$
\mathcal{C}_{\delta}=\{z \in \mathbf{C}:|z-\nu| \leq \delta, \nu \in[\alpha, \beta]\}
$$

Then, $d_{\delta_{0}}>0$ for some $\delta_{0}>0$.

Recall that $s^{z}$ is analytic on $\mathcal{R}_{0}$ for every fixed $z>0$. Hence, $(j \omega)^{z}$ is analytic on $\mathcal{R}_{0}$ for every nonzero point $j \omega$ on the imaginary axis.

Proof. Write $\mu$ in the polar coordinates as $\mu=r e^{j \varphi}$ where from (6) $\varphi$ satisfies $|\varphi|<\pi\left(1-\frac{\beta}{2}\right)$. Assume first $\varphi \geq 0$ and consider the infimum in (13) over $\mathbf{R}_{-}$instead of $\mathbf{R}$. Let $z=\xi+j \eta \in \mathcal{C}_{\delta}$. Then, for all $\delta, \omega>0$

$$
\begin{aligned}
\left|(-j \omega)^{z}+\mu\right|^{2}= & \left|e^{z\left(\ln \omega-j \frac{\pi}{2}\right)-j \varphi}+r\right|^{2} \\
= & \left|\omega^{\xi} e^{\frac{\pi \eta}{2}} e^{-j\left(\frac{\pi \xi}{2}+\varphi-\eta \ln \omega\right)}+r\right|^{2} \\
= & \omega^{2 \xi} e^{\pi \eta}+r^{2} \\
& +2 r \omega^{\xi} e^{\frac{\pi \eta}{2}} \cos \left(\frac{\pi \xi}{2}+\varphi-\eta \ln \omega\right) .
\end{aligned}
$$


Now, assume $\delta \leq \frac{\alpha}{4}$ and let $\Omega=\max \left\{(2 r e)^{\frac{4}{3 \alpha}}, 1\right\}$. Then, for all $\omega \geq \Omega$ and $z \in \mathcal{C}_{\delta}$ with $\delta \leq \frac{\alpha}{4}$ from the second equality in (15) we have

$$
\left|(-j \omega)^{z}+\mu\right| \geq\left|\omega^{\xi} e^{\frac{\pi \eta}{2}}\right|-r \geq r
$$

It remains to study the case $\omega<\Omega$. Choose a $\delta_{0}>0$ such that

$$
\kappa\left(\delta_{0}\right)=\frac{\pi \beta}{2}+\varphi+\delta_{0} \ln \Omega<\pi
$$

which is possible since $\frac{\pi \beta}{2}+\varphi<\pi$. Without loss of generality, we assume $\frac{\pi \beta}{2}+\varphi \geq \frac{\pi}{2}$. Otherwise, pick a $\delta_{0} \leq \frac{\alpha}{4}$ satisfying $\kappa\left(\delta_{0}\right) \leq \frac{\pi}{2}$; and for all $z \in \mathcal{C}_{\delta_{0}}$ and $\omega \leq \Omega$, from the last equality in $(15)$ we get $\left|(-j \omega)^{z}+\mu\right| \geq r$. Thus,

$$
\left|(-j \omega)^{z}+\mu\right|^{2} \geq \omega^{2 \xi} e^{\pi \eta}+r^{2}-2 r \omega^{\xi} e^{\frac{\pi \eta}{2}} \cos \zeta .
$$

where $\zeta=\pi-\kappa\left(\delta_{0}\right)$. Note that $0<\zeta \leq \frac{\pi}{2}$. The righthand side of the inequality in (17) is a quadratic function of $\omega^{\xi} e^{\frac{\pi \eta}{2}}$. Its minimum is achieved at $\omega^{\xi} e^{\frac{\pi \eta}{2}}=r \cos \zeta$. It follows that for all $\omega \leq \Omega$ and $z \in \mathcal{C}_{\delta_{0}}$,

$$
\left|(-j \omega)^{z}+\mu\right| \geq r \sin \zeta .
$$

Combining (16) and (18), we obtain $d_{\delta_{0}}>0$ when $\varphi \geq$ 0 and the infimum in (13) is restricted to $\mathbf{R}_{-}$. The inequalities (16) and (18) with $-j w$ replaced by $j \omega$ and $\eta$ replaced by $-\eta$ still hold when the infimum in (13) is restricted to $\mathbf{R}_{+}$. Hence, $d_{\delta_{0}}>0$ for some $\delta_{0}>0$ whenever $\varphi \geq 0$. The proof of the case $\varphi<0$ follows by taking the complex conjugate of (13) and noting that $\mathcal{C}_{\delta}$ is symmetric with respect to the real axis.

Proposition 4.1. Given two numbers $\alpha, \beta \in(0,2)$ with $(\beta, \mu)$ satisfying $(6)$, pick a nonnegative integer $m$ such that

$$
2(m \gamma+\alpha)>1 \text {. }
$$

Then, there exists a $\delta^{*}>0$ such that for all $h \in H_{2}(\Pi)$,

$$
\psi_{h}(z)=\int_{-\infty}^{\infty} \frac{\overline{h(j \omega)} d \omega}{\left[(j \omega)^{\gamma}+\lambda\right]^{m}\left[(j \omega)^{z}+\mu\right]}, \quad z \in \mathcal{C}_{\delta^{*}}
$$

defines analytic functions on $\mathcal{C}_{\delta^{*}}$.

Proof. From Lemma 1, we have $d_{\delta_{0}}>0$ for some $\delta_{0}>0$ whenever $(\beta, \mu)$ satisfies (6). Thus, for all $h \in H_{2}(\Pi)$, the functions in (20) are well-defined provided that $m$ is chosen sufficiently large. In fact, from an application of the Cauchy-Schwarz inequality, we get

$$
\left|\psi_{h}(z)\right|^{2} \leq\|h\|_{2}^{2} \int_{-\infty}^{\infty} \frac{d \omega}{\left|(j \omega)^{\gamma}+\lambda\right|^{2 m}\left|(j \omega)^{z}+\mu\right|^{2}}
$$

where the integrand of the second factor on the righthand side of the inequality in (21) is a uniformly bounded function on $\mathbf{R} \times \mathcal{C}_{\delta_{0}}$. Hence, by letting $m^{*}$ to satisfy the inequality $2\left[m^{*} \gamma+\operatorname{Re}(z)\right]>1$, we conclude that the integrals in (21) are finite for all $m \geq m^{*}$. In order to fix $m^{*}$ over $\mathcal{C}_{\delta_{0}}$, it suffices to let $2\left(m^{*} \gamma+\alpha-\delta_{0}\right)>1$. Now, if an $m$ satisfying $2(m \gamma+\alpha)>1$ is given, then pick a $\delta^{*}$ satisfying the inequalities $0<\delta^{*} \leq \delta_{0}$ and $2\left(m \gamma+\alpha-\delta^{*}\right)>1$.

An application of the bounded convergence theorem to (20) shows that $\psi_{h}(z)$ is continuous on $\mathcal{C}_{\delta^{*}}$. Next, we apply the Morera's theorem (see, for example, Theorem 10.17 in Rudin (1987)) to show that $\psi_{h}(z)$ is analytic on $\mathcal{C}_{\delta^{*}}$. In the application of the Morera's theorem, the change of the integration orders is justified by the Fubini's theorem, and the fact that $\left[(j \omega)^{z}+\mu\right]^{-1}$ is analytic on $\mathcal{C}_{\delta^{*}}$ is used.

The essence of Proposition 4.1 is the fact that the functions defined by $(20)$ are analytic on some domain $\mathcal{C}_{\delta^{*}}$ that contains the closed interval $[\alpha, \beta]$. Now, we are prepared to establish the main result of this paper.

Let

$$
\underline{\gamma}=\inf _{k} \gamma_{k}, \quad \bar{\gamma}=\sup _{k} \gamma_{k}
$$

and assume that $0<\gamma<\bar{\gamma}<2$. Clearly, it is sufficient to consider the spanning properties of the functions $\phi_{k 1}(z)$, $k \geq 1$. The main result is summarized in the following.

Theorem 4.2. Consider the generator functions (7) with $\gamma_{k}$ lying in a compact subset $[\underline{\gamma}, \bar{\gamma}]$ of the interval $(0,2)$. Assume that $(\gamma, \lambda)$ and $(\bar{\gamma}, \mu)$ satisfy $(6)$. Pick a nonnegative integer $m$ satisfying $2(m \gamma+\underline{\gamma})>1$ and set $m_{k}=m$ for all $k$. Then, $\phi_{k \ell}, \ell=1, \cdots, n_{k} ; k \geq 1$ are complete in $\mathrm{H}_{2}(\Pi)$ if and only if

$$
\phi_{\nu 1}(s)=\frac{1}{\left(s^{\gamma}+\lambda\right)^{m}\left(s^{\nu}+\mu\right)}, \quad \nu \in[\underline{\gamma}, \bar{\gamma}]
$$

are complete in $H_{2}(\Pi)$.

Proof. Assume that there exists a nontrivial $h \in H_{2}(\Pi)$ orthogonal to all functions $\phi_{k 1}$ :

$$
\int_{-\infty}^{\infty} \frac{\overline{h(j \omega)} d \omega}{\left[(j \omega)^{\gamma}+\lambda\right]^{m}\left[(j \omega)^{\gamma_{k}}+\mu\right]}=0, \quad k=1,2, \cdots
$$

which implies from (20) that

$$
\psi_{h}\left(\gamma_{k}\right)=0, \quad k=1,2, \cdots .
$$

Recall that $\psi_{h}(z)$ is analytic on $\mathcal{C}_{\delta^{*}}$ for some $\delta^{*}>0$. Moreover, (25) tells also that the zeros of $\psi_{h}(z)$ has an accumulation point in $\mathcal{C}_{\delta^{*}}$ since $\gamma_{k} \in[\underline{\gamma}, \bar{\gamma}] \subset \mathcal{C}_{\delta^{*}}$ for all $k$. This is possible only if $\psi_{h}(z)$ vanishes on $\mathcal{C}_{\delta^{*}}$, i.e., $\psi_{h}(z)=0$ for all $z \in \mathcal{C}_{\delta^{*}}$. In particular,

$$
\int_{-\infty}^{\infty} \frac{\overline{h(j \omega)} d \omega}{\left[(j \omega)^{\gamma}+\lambda\right]^{m}\left[(j \omega)^{\nu}+\mu\right]}=0, \text { for all } \nu \in[\underline{\gamma}, \bar{\gamma}]
$$

Hence, $\phi_{k 1}, k=1,2, \cdots$ are not complete in $H_{2}(\Pi)$ if and only if the linear span of the functions defined in (23) is not dense in $H_{2}(\Pi)$.

If it is possible to construct a complete basis for $H_{2}(\Pi)$ in the form (7), then Proposition 4.2 generates uncountably many others of the same type by simply varying the orders of the fractional derivatives between the two extreme values. Furthermore, it is sufficient to check the completeness of the uncountable set $\phi_{\nu 1}(s), \nu \in[\gamma, \bar{\gamma}]$. Likewise, the question of impossibility of constructing a complete basis for $\mathrm{H}_{2}(\Pi)$ in the form (7) can be studied by checking whether the latter infinite set is complete or not. 


\section{CONCLUSIONS}

In this paper, completeness problem for a special class of incommensurable fractional basis functions was studied. It was established that the completeness problem for this class is equivalent to another completeness problem for a particular set of uncountably many basis functions. This equivalence is expected to simplify the ensuing analysis. The study of the latter problem remains as a future work.

\section{REFERENCES}

H. Akçay. Synthesis of complete orthonormal fractional basis functions with prescribed poles. In Proceedings of the 7th IFAC Symposium on Nonlinear Control Systems, Pretoria, South Africa, August 2007.

H. Akçay and B. Ninness. Orthonormal basis functions for modelling continuous-time systems. Signal Processing, 77:261-274, 1999a.

H. Akçay and B. Ninness. Orthonormal basis functions for continuous-time systems and $L_{p}$ convergence. Mathematics of Control, Signals, and Systems, 12:295-305, 1999b.

M. Aoun, R. Malti, F. Levron, and A. Oustaloup. Synthesis of fractional Laguerre basis for system approximation. Automatica, 43:1640-1648, 2007.

J. L. Battaglia, O. Cois, L. Puigsegur, and A. Oustaloup. Solving an inverse heat conduction problem using a noninteger identified model. International Journal of Heat and Mass Transfer, 44:2671-2680, 2001.

A. Benchellal, T. Poinot, and J. C. Trigeassou. Advances in fractional calculus. Theoretical developments and applications in physics and engineering, chapter Modelling and identification of diffusive systems using fractional models, pages 213-225. Springer, 2007. edited by J. Sabatier and O. M. Agrawal and J. A. Tenreiro Machado.

H. W. Bode. Network Analysis and Feedback Amplifier Design. Van Nostrand, New York, 1945.

C. Bonnet and J. R. Partington. Coprime factorizations and stability of fractial differential systems. Systems 8 Control Letters, 41:167-174, 2000.

B. Epstein. Orthogonal Families of Analytic Functions. Macmillan, New York, 1965.

P. Heuberger, P. M. J. Van den Hof, and O. Bosgra. A generalized orthonormal basis for linear dynamical systems. IEEE Transactions on Automatic Control, 40: 451-465, 1995.

N. Heymans and J. C. Bauwens. Fractal rheological models and fractional differential equations for viscoelastic behavior. Rheologica Acta, 33:219, 1994.

L. Le Lay, A. Oustaloup, and J. C. Trigeassou. Frequency identification by implicit derivative models. In $A V C S$ '98 International Conference on Advances in Vehicle Control and Safety, pages 351-356, Amiens, France, 1998.

J. Liouville. Mémoire sur quelques questions de géométrie et de mécanique. J. Ecole Polytech, 13:1-69, 1832.

R. Malti, M. R. Aoun, and A. Oustaloup. Synthesis of fractional Kautz-like basis with two periodically repeating complex conjugate modes. In International Symposium on Control, Communications and Signal Processing, ISCCSP, pages 835-839, 2004.

R. Malti, M. Aoun, J. Sabatier, and A. Oustaloup. Tutorial on system identification using fractional differentiation models. In $S Y S I D$, pages 606-611, Newcastle, Australia, 2006.

B. Mandelbrot and J. W. Van Ness. Fractional Brownian motions, fractional noises and applications. SIAM Review, 10, 1968

D. Matignon. Stability properties for generalized fractional differential systems. In ESAIM proceedings - Systèmes Différentiels Fractionnaires - Modèles, Méthodes et Applications, volume 5, pages 145-158, 1998.

D. Matignon and B. D'Andrea-Novel. Some results on controllability and observability of finite-dimensional fractional differential systems. In Computational Engineering in Systems Applications, volume 2, pages 952956, 1996. IMACS, IEEE-SMC.

J. Mendel. A unified approach to the synthesis of orthonormal exponential functions useful in systems analysis. IEEE Trans. Systems Sci. Cybernet., 2:54-62, 1966.

B. Ninness, H. Hjalmarsson, and F. Gustafsson. Generalized Fourier and Toeplitz results for rational orthormal bases. SIAM J. Control Optim., 37:429-460, 1998.

K. B. Oldham and J. Spanier. The replacement of Fick's laws by a formulation involving semi-differentiation. Electro-anal. Chem. Interfacial Electrochem, 26:331341, 1970.

K. B. Oldham and J. Spanier. A general solution of the diffusive equation for semiinfinite geometries. Journal of Mathematical Analysis and Applications, 39:655-669, 1972

K. B. Oldham and J. Spanier. Diffusive transport to planar, cylindrical and spherical electrodes. Electroanaly. Chem. Interfacial Electrochem., 41:351-358, 1973.

K. B. Oldham and J. Spanier. The Fractional Calculus. Academic Press, New York, 1974.

A. Oustaloup and B. Mathieu. La commande CRONE : du scalarie au multivariable. Paris, 1999. Hermes.

I. Podlubny. Fractional-order systems and PID-controllers. IEEE Trans. Automat. Contr., 44:208-214, 1999.

B. Riemann. Gesammelte Werke. 1892.

S. Rodrigues, N. Munichandraiah, and A. K. Shukla. A review of state of charge indication of batteries by means of A.C. impedance measurements. Journal of Power Sources, 87:12-20, 2000.

W. Rudin. Real and Complex Analysis. McGraw-Hill, New York, 1987. Third edition.

J. Sabatier, M. Moze, and A. Oustaloup. On fractional systems $H_{\infty}$-norm computation. In In Proc. 44th IEEE Conference on Decision and Control, and the European Control Conference, pages 5758-5763, Seville, Spain, 2005

S. G. Samko, A. A. Kilbas, and O. I. Marichev. Fractional Integrals and Derivatives: Theory and Applications. Gordon and Breach Science, Singapore, 1993.

V. R. Schneider. Fractional diffusion. In Proc. Dynamics Stochastic Process, Theory and Applications, pages 276286, 1990. Workshop.

V. Vorperian. A fractal model of anomalous losses in ferromagnetic materials. In PESC'92 Rec. 23rd Annual IEEE Power Electronics Specialists Conf., volume 2, pages 1277-1283, 1992.

B. Wahlberg and P. M. Mäkilä. On approximation of stable linear dynamical systems using Laguerre and Kautz functions. Automatica, pages 693-708, 1996. 